\title{
EFFECTIVE IMPLEMENTATION OF GENERIC MARKET MODELS
}

\author{
BY
}

\author{
MARK S. Joshi AND LOREnZO LIESCH
}

\begin{abstract}
A number of standard market models are studied. For each one, algorithms of computational complexity equal to the number of rates times the number of factors to carry out the computations for each step is introduced. Two new classes of market models are developed and it is shown for them that similar results hold.
\end{abstract}

\section{KEYWORDS}

Market model, efficiency, interest rate derivatives.

\section{INTRODUCTION}

It has become more common in recent years for insurance companies to invest their reserves in exotic structured products in order to obtain an increase in yield. Such products typically consist of a note issued by a bank which pays a coupon which is a path-dependent function of LIBOR or swap rates. This note is often callable, that is the bank can repay the principal early and terminate the contract. It is therefore important to be able to accurately assess the value of these products.

Methodologies for pricing such interest-rate products have evolved over time. This evolution both reflects the changing nature of the products being addressed and technical advances: both mathematical and computational. A recent development is the introduction of generic market models. In this article, we discuss their context in the evolution of interest rate models, and introduce some new algorithms demonstrating that their efficiency level is comparable to that of the LIBOR market model.

The post Black-Scholes models for pricing interest rate derivatives were initially short rate models. These models relied on the notion of a hypothetical short rate chosen to follow a process that was typically some variant of a normal or log-normal process. One can view exotic options pricing as an extrapolation exercise, the objective is to extrapolate from the prices of vanilla derivative instruments such as swaps and bonds, and options thereon, to prices 
of exotic derivatives. One therefore had to fit the short rate process's parameters to the prices of market instruments. In particular, calibrating to both the discount curve and the prices of swaptions were non-trivial tasks. A consequence of all this fitting and the fact that everything was driven by a single short rate was that the dynamics of the model were not realistic. The interest rate discount curve can evolve in a complicated fashion which is not capturable by a single state variable.

Market models were a significant advance on short rate models. These models rely on choosing processes for market observable rates rather than a hypothetical short rate. The rates evolved are typically LIBOR rates or swaprates and one then has automatic calibration not just to the value of the rate but also to options on that rate; that is caplets and swaptions. The original cases studied were contiguous LIBOR rates and co-terminal swap rates, (Brace et al, 1997, Jamshidian, 1997) however, more general cases have recently been examined also, (Gallucio et al 2007, Gallucio and Hunter 2004, Pietersz and Regenmortel 2006), including the concept of a generic market model. For detailed accounts of market models, see Brigo and Mercurio (2001), Joshi (2003) or Musiela and Rutkowski (1997).

In all of these models, $n$ rates are chosen and assumed to be log-normal (or some similar process such as displaced diffusion) and a single zero-coupon bond is chosen as numeraire. The fact that rates are log-normal and evolved individually means not only that calibration to their initial values is immediate; but also that calibration to options on their values is also by construction rather than fitting. We can thus price $n$ swaptions of our choice absolutely correctly with no work, and additional swaptions can be calibrated to with a fitting procedure. Contrast this with a short-rate model where even achieving a fit to $n$ swaptions requires an optimization. The fact that we can choose these swaptions is an important advantage when pricing exotic interest rate derivative products in that it allows us to choose the $n$ rates which have the closest relationship with its pay-off.

To price exotic derivatives, one typically evolves the rates through a series of dates. On each date, the cash-flows generated by a product are computed and used to purchase units of the numeraire. One then gathers the total number of numeraires generated by each path, and averages to get an estimate of the expected pay-off as a multiple of the initial value of the numeraire.

There will generally be $n$ rates driven by an $F$-dimensional Brownian motion, and this will be said to be an $F$-factor model. Generally, in derivatives pricing one works with discounted (or deflated) asset prices which are constructed to be martingales in an appropriate measure. This is, however, not natural for market models since the evolved quantities are rates, i.e., ratios of asset prices. In the pricing measure, the rates therefore have drifts; the values of these drifts are determined by the rates and the covariances between them. This means that the implementation of a pricing model by Monte Carlo is tricky and computationally intensive. The problem of implementation of swap-rate market models by means other than Monte Carlo does not appear to have been addressed. 
The implementation of such models requires several non-trivial computations. The first is the deduction of bond ratios from the observed rates, the second is the calculation of drifts, and thirdly the stochastic differential equation must be approximately solved. In this paper, we address how all of these can be done with a total of order $n F$ computations for a wide range of cases including all the specific examples that have been studied.

In particular, we study the cases of co-terminal swap-rates, co-initial swap rates and the constant maturity market model. We also introduce two more general types of model: the incremental market model and the fully incremental market model, and establish similar results for them.

Specifically, we find order $n$ algorithms for the deduction of the bond ratios in each of our specific cases, and for fully incremental models. We also show that the drifts computation is order $n F$ in each of these cases. The simple Euler approximation (and also predictor corrector) is of order $n F$ for solving the SDEs so the total computational order for a step in all these cases is therefore $n F$.

It is important to realize that it is not necessary to find a closed-form formula for the drift and the bond ratios, but merely to write down an algorithm that is efficiently implementable, and this is how we proceed.

The only papers to date where the issue of efficient algorithms for the computation of swap-rate drifts and the deduction of bond ratios are discussed are Joshi (2003b, 2006) and Pietersz and Regenmortel (2006). In Joshi (2003b), the LIBOR market model is studied and an algorithm for drift computation of order $n F$ is presented. In Joshi (2006), this result is extended to encompass the case of $F$ common factors and $n$ idiosynchratic factors. Note that a similar extension could easily carried out in the cases studied here, but we do not so for brevity. In Pietersz and Regenmortel (2006), an order $n^{3}$ algorithm in presented for deduction of bond ratios in the general case. An order $n F$ algorithm is presented for computation of drifts in the co-terminal case, and an approximate algorithm for the drifts of order $n F$ is presented for the constant maturity case.

The structure of this paper is as follows. In Section 2, we establish some notation and examine the computational order of the evolution of the SDE. We develop computational techniques in Section 4, which we apply in the rest of the paper. In Section 5, we show how to deduce the bond ratios from the swap rates in the co-terminal model, and the drift computation in that case is carried out in Section 6. The constant maturity market model is dealt with in Section 7. The arguments for the co-initial model are developed in Section 8. The concept of an incremental market model is introduced in Section 9. We present some numerical results in Section 10 and conclude in Section 11.

\section{NOTATION AND MODEL SET-UP}

We fix some notation. We have times $0<t_{0}<t_{1}<\cdots<t_{n}$, and $\tau_{j}=t_{j+1}-t_{j}$. We let $P_{j}$ denote the price of the zero-coupon bond expiring at time $t_{j}$. We let $\mathrm{SR}_{\alpha, \beta}$ 
denote the swap-rate running from time $t_{\alpha}$ to time $t_{\beta}$. We let $A_{\alpha, \beta}$ be the value of the annuity of $\mathrm{SR}_{\alpha, \beta}$, that is

$$
A_{\alpha, \beta}=\sum_{j=\alpha}^{\beta-1} \tau_{j} P_{j+1} .
$$

Recall, that the swap-rate is the fixed rate at which a stream of cash-flows can be swapped to a floating rate for zero cost. It satisfies

$$
\mathrm{SR}_{\alpha, \beta}=\frac{P_{\alpha}-P_{\beta}}{A_{\alpha, \beta}} .
$$

A forward rate corresponds to the degenerate case where $\beta=\alpha+1$.

In all market models, one takes $n$ swap-rates and assigns dynamics to them. This is done in such a way as to make the ratio of all the bonds to the numeraire asset $P_{N}$ be martingales. Note that $n$ is precisely the number of rates needed to deduce the values of the $n$ ratios $P_{j} / P_{N}$.

Picking a swap-rate is equivalent to picking a pair $\left(\alpha_{j}, \beta_{j}\right)$ with $\beta_{j} \geq \alpha_{j}+1$, so specifying a generic market model is equivalent to picking $n$ such pairs. Having done so, we work with the rates

$$
\mathrm{SR}_{j}=\mathrm{SR}_{\alpha_{j}, \beta_{j}} .
$$

Clearly, one cannot pick an arbitrary set of pairs $\left(\alpha_{j}, \beta_{j}\right)$. For example, for every $i$ one would expect there to be at least one swap-rate that either terminates or starts at $i$ in order to determine the ratio $P_{i} / P_{N}$. The issue of what choices lead to self-consistent models has been addressed by Gallucio et al (2007), and Pietersz and Regenmortel (2006). In particular, Gallucio et al used graphtheoretic techniques to show that there are $n^{n-2}$ possible market models. Given that $n$ is usually at least 20, this leads to a huge number of possible choices.

However, as well as it being possible to deduce the ratios, one also needs an effective algorithm for doing so, since this will be carried out at every step of every path in a Monte Carlo simulation. Pietersz and Regenmortel showed that in general it was possible to carry out the deduction with order $n^{3}$ computations. Whilst this is a useful result, for $n$ large it can be very time consuming and one generally wants a faster algorithm. We show in this paper that for large sub-classes of generic market models, an order $n$ algorithm exists.

When pricing in a market model, one carries out a Monte Carlo simulation to estimated the expected discounted value of a pay-off. Each path will consist of a number of steps and for each step the following procedures are necessary:

(1) Compute the drifts of the rates.

(2) Evolve the rates.

(3) Deduce the bond ratios.

(4) Compute cash-flows generated by the product.

(5) Discounting the cash-flows. 
The last two of these are of constant complexity for any reasonable product. Since the third is of linear complexity, we would therefore also like the first two to be of linear complexity, we will show that this can be made to be the case for a large class of models.

The rates will be driven by $F$ Brownian motions. Let $\tilde{a}_{j k}$ denote the exposure of rate $j$ to the Brownian motion $W_{k}$. We will therefore have a system of stochastic differential equations,

$$
d \mathrm{SR}_{j}=\tilde{\mu}_{j}(\mathrm{SR}) d t+\mathrm{SR}_{j} \sum_{k=1}^{F} \tilde{a}_{j k}(t) d W_{k}
$$

We will evolve the log in discrete time steps. A typical step will run from $s$ to $t$, for some $s<t$, and then we will have

$$
\log \mathrm{SR}_{j}(t)=\log \mathrm{SR}_{j}(s)+\mu_{j}-\sum_{k=1}^{F} a_{j k} Z_{k},
$$

where $a_{j k}$ is the integral of $\tilde{a}_{j k}$ across the step, $C_{j j}$ is the variance of $\log \mathrm{SR}_{j}$ and $Z_{k}$ are $F$ independent random variables.

The terms $a_{j k}$ specify the volatilities and covariance of the rates: choosing them is equivalent to calibrating the model.

We have also set $\mu_{j}$ to be the approximated drift across the step. In this paper, we will take it to be instantaneous drift at the start of the step with the covariance terms across the step integrated. Given the drift term, it is clear that the evolution across the step could be carried out with order $n F$ computations.

A predictor-corrector drift approximation method is often used to better approximate the evolution of the SDE. In this case, the rates are first evolved as above, and then the drifts are recomputed at the end of the step. The rates are then re-evolved using the same random numbers but with the average of the two drifts. This method was introduced in Hunter et al (2001). Clearly, if drifts can be deduced in order $n F$ computations, then the predictor-corrector method can also be applied with the same computational order.

\section{A SIMPLE CASE}

In this section, we discuss the LIBOR market model. The results are not original but they serve to illustrate the issues and will hopefully assist the reader with the rest of the paper, see Joshi (2003b) for further discussion of this case. The LIBOR market model corresponds to the case where all swap rates span one period, so the rates are

$$
f_{j}=\mathrm{SR}_{j, j+1} .
$$


In this case, we have

$$
\frac{P_{j}}{P_{j+1}}=1+f_{j} \tau_{j}
$$

so it is easy to deduce the bond ratios from the rates.

If we take the final bond as numeraire, then we want the measure in which every bond divided by the numeraire is a martingale. This makes the rates have non-zero drifts. It is a well-known result that the processes followed by the rates in this measure are

$$
\frac{d f_{j}}{f_{j}}=\mu_{j}(f) d t+\sum_{k=1}^{F} C_{j k} d W_{k},
$$

where

$$
\mu_{j}=-\sum_{r=j+1}^{n} C_{j r} \frac{f_{r} \tau_{t}}{1+f_{r} \tau_{r}}
$$

with $C_{j r}=\rho_{j r} \sigma_{j} \sigma_{r}$, the instantaneous covariance between the logs of rates $r$ and $j$. (See for example Brigo and Mercurio (2001) or Joshi (2003).)

To evolve the log rates across a step, one needs to compute $\mu_{j}$ for each $j$. We pre-compute the ratios $\frac{f_{r} \tau_{t}}{1+f_{r} \tau_{r}}$. The computation of $\mu_{j}$ then needs roughly $n-j$ multiplications. So computing the value for all $j$, will require order $n^{2}$ computations. If the number of factors is low and $n$ is large, then the drift computation will dominate computing time.

However, if the number of factors is $F$, we can write

$$
C=A A^{t},
$$

with $A=\left(a_{i j}\right)$ an $n \times F$-dimensional matrix. This will allow us to rewrite the computation in a manner that allows a timing of order $n F$. In particular, substituting for the value of $C_{j k}$ and reversing the order of summation, we obtain

$$
\mu_{j}=-\sum_{r=1}^{F} a_{j r} e_{j, r},
$$

where

$$
e_{j, r}=\sum_{k>j} a_{k r} \frac{f_{k} \tau_{k}}{1+f_{k} \tau_{k}} .
$$

The virtue of this representation is that

$$
e_{j, r}=e_{j+1, r}+a_{j+1, r} \frac{f_{j+1} \tau_{j+1}}{1+f_{j+1} \tau_{j+1}},
$$


which means we can obtain the value of $e_{j, r}$ for all $j$ and $r$ with order $n F$ computations. The deduction of the terms $\mu_{j}$ then requires another $n F$ computations and the result follows.

Whilst in the LIBOR market model case, it is easy to get closed-form formulas which can then be manipulated to get faster computational time, for more general swap-rate market models one has the issue that the drifts are not easily writable as formulas. Instead, they can be more naturally found as results of recursive procedures. Our objective in this paper is therefore to show how these recursive procedures can be quickly evaluated.

\section{THE CROSS-VARIATION DERIVATIVE AND MARTINGALE PRICING}

In what follows, it will be useful to work with the cross-variation derivative for two Ito processes. Given processes $X_{t}$ and $Y_{t}$, we define

$$
\left\langle X_{t}, Y_{t}\right\rangle
$$

to be the coefficient of $d t$ in $d X_{t} . d Y_{t}$. (Note the cross-variation process is generally defined to be the process $d X_{t} \cdot d Y_{t}$, but the cross-variation derivative will be more convenient for us.) This means that if we have

$$
\begin{gathered}
d X_{t}=\mu_{X}(t) d t+\sigma_{X}\left(X_{t}, Y_{t}, t\right) d W_{t}^{X}, \\
d Y_{t}=\mu_{Y}(t) d t+\sigma_{Y}\left(X_{t}, Y_{t}, t\right) d W_{t}^{Y},
\end{gathered}
$$

with $W^{X}, W^{Y}$ correlated (jointly normal) Brownian motions, then

$$
\left\langle X_{t}, Y_{t}\right\rangle=\rho \sigma_{X}\left(X_{t}, Y_{t}, t\right) \sigma_{Y}\left(X_{t}, Y_{t}, t\right),
$$

where $\rho$ is the correlation between $W^{X}$ and $W^{Y}$. Note that the drift terms do not appear in this expression.

The cross-variation derivative has some useful computational properties. First, it is linear in each term, i.e, if $Y_{j}$ are a number of stochastic processes, and $\alpha_{j} \in \mathbb{R}$, then

$$
\left\langle X, \sum_{j} \alpha_{j} Y_{j}\right\rangle=\sum_{j} \alpha_{j}\left\langle X, X_{j}\right\rangle .
$$

It is trivially symmetric in $X$ and $Y$. We can also compute with products in a simple fashion

$$
\langle X, Y Z\rangle=\langle X, Y\rangle Z+\langle X, Z\rangle Y .
$$

Note that trivially the cross-variation derivative with a constant is always zero. 
We can deduce the value of $\left\langle Y, X^{-1}\right\rangle$ :

$$
\begin{aligned}
\langle Y, 1\rangle & =\left\langle Y, X . X^{-1}\right\rangle, \\
& =\langle Y, X\rangle X^{-1}+\left\langle Y, X^{-1}\right\rangle X,
\end{aligned}
$$

and therefore

$$
\left\langle Y, X^{-1}\right\rangle=-X^{-2}\langle Y, X\rangle .
$$

The cross-variation derivative will play an important role in our drift computations. Suppose we have a rate, by which we shall mean a quantity defined as the ratio of the prices of two assets; in other words, it is the exchange rate for converting one asset to the other. When applying martingale pricing, we pick a numeraire asset $N$ which is always positive, and construct a measure in which the ratio of the price of every security to $N$ is a martingale. We can think of $N$ as a deflator. Once everything is a martingale, no arbitrage can occur in either the pricing measure, nor any measure equivalent to it. Whilst traditionally, one started by positing real-world processes and finding the equivalent martingale measure, it is now more usual to start by specifying dynamics in the martingale measure.

So suppose $X, Y$ and $N$ are tradable assets, and we wish to compute the drift of $R=X / Y$ when $N$ is numeraire. We want $R Y / N=X / N$ and $Y / N$ to be martingales. Up to technical considerations, we do not address here, this is equivalent to their drifts being zero. We compute to find the drift of $R$ which makes this the case.

We have

$$
d \frac{R Y}{N}=\frac{Y}{N} d R+R d \frac{Y}{N}+d R \cdot d \frac{Y}{N} .
$$

Taking the drifts, and discarding martingale terms, we have that $\mu_{R}$, the drift of $R$, satisfies

$$
\mu_{R}=-\frac{N}{Y}\left\langle R, \frac{Y}{N}\right\rangle .
$$

So, in order to compute the drift of $R$, it is sufficient to compute the cross-variation derivative of $R$ and $\frac{Y}{N}$.

The cross-variation will also be useful for assessing the impact of changing numeraire on a drift. Suppose we already know the drift, $\mu_{R, N}$, of $R$ under the numeraire $N$ and we want to compute the drift, $\mu_{R, M}$, with numeraire $M$. We have

$$
\begin{aligned}
\mu_{R, M} & =-\left\langle R, \frac{Y}{M}\right\rangle \frac{M}{Y}, \\
& =-\left\langle R, \frac{Y}{N} \frac{N}{M}\right\rangle \frac{M}{Y},
\end{aligned}
$$




$$
\begin{aligned}
& =-\left\langle R, \frac{Y}{N}\right\rangle \frac{N}{M} \frac{M}{Y}-\left\langle R, \frac{N}{M}\right\rangle \frac{Y}{N} \frac{M}{Y}, \\
& =\mu_{R, N}-\left\langle R, \frac{N}{M}\right\rangle \frac{M}{N} .
\end{aligned}
$$

We now do the computation using cross-variation derivatives in the LIBOR market model case with the terminal measure (that is with $P_{n}$ as numeraire.) Since

$$
f_{j}=\frac{P_{j}-P_{j+1}}{\tau_{j} P_{j+1}},
$$

the drift of $\mu_{j}$, is

$$
\begin{aligned}
-\frac{P_{n}}{P_{j+1}}\left\langle f_{j} \frac{P_{j+1}}{P_{n}}\right\rangle & =-\frac{P_{n}}{P_{j+1}} \sum_{k=1}^{F} f_{j} \tilde{a}_{j k}\left\langle W_{k}, \frac{P_{j+1}}{P_{n}}\right\rangle, \\
& =-\frac{P_{n}}{P_{j+1}} \sum_{k=1}^{F} f_{j} \tilde{a}_{j k}\left\langle W_{k}, \prod_{r=j+1}^{n} \frac{1}{1+f_{r} \tau_{r}}\right\rangle, \\
& =-\frac{P_{n}}{P_{j+1}} \sum_{k=1}^{F} \sum_{r=j+1}^{n} f_{j} \tilde{a}_{j k} \frac{P_{j+1}}{P_{n}}\left(1+f_{r} \tau_{r}\right)\left\langle W_{k}, \frac{1}{1+f_{r} \tau_{r}}\right\rangle, \\
& =-f_{j} \sum_{k=1}^{F} \tilde{a}_{j k} \sum_{r=j+1}^{n}\left(1+f_{r} \tau_{r}\right)^{-1}\left\langle W_{k}, 1+f_{r} \tau_{r}\right\rangle, \\
& =-f_{j} \sum_{k=1}^{F} \tilde{a}_{j k} \sum_{r=j+1}^{n} \frac{\tilde{a}_{k r} \tau_{r} f_{r}}{1+f_{r} \tau_{r}} .
\end{aligned}
$$

Thus we have achieved an alternate deduction of the drift expression in this simple case.

\section{Deducing the bond-Ratios In the Co-Terminal CASE}

The most ancient and most popular swap-rate market model is the co-terminal model of Jamshidian (1997). In this model, the swap rates are chosen to all have the same termination date but varying start dates. This model is particularly well adapted to the pricing of Bermudan swaptions. Recall that a Bermudan swaption is the right to enter into a swap on any of a fixed set of dates. A callable bond can be viewed as a fixed rate non-callable bond minus a Bermudan swaption. If one restricts exercise to any one of these dates then one has a European swaption, that is a swaption that can only be exercised on one date. The co-terminal model has the attractive feature that calibration to these swaptions is immediate. 
In this section, we show how to compute the bond ratios in order $n$ computations for the co-terminal swap-rate market model. In order to keep notation simple, in this section and the next, we let $\mathrm{SR}_{j}$ denote the swap-rate associated to times $t_{j}, \cdots, t_{n}$. We also let $A_{j}$ be the annuity of $\mathrm{SR}_{j}$.

We first show how to find the ratios $P_{j} / P_{n}$. Clearly, the ratio $P_{j} / P_{N}$ for any $N$ is then trivial to find. We work backwards. If $j=n$, we have $P_{j} / P_{n}=1$, and we are done. For $j<n$, we assume that $P_{j} / P_{n}$ has been found for larger $j$. We then have

$$
\mathrm{SR}_{j}=\frac{P_{j}-P_{n}}{A_{j}}
$$

and it follows that

$$
\frac{P_{j}}{P_{n}}=1+\mathrm{SR}_{j} \frac{A_{j}}{P_{n}}
$$

The terms on the right hand side are already determined as $A_{j}$ only involves bonds with maturity after $t_{j}$, the value of $P_{j} / P_{n}$ follows and we are done.

Note that all the bond ratios can be deduced with order $n$ computations.

\section{CO-TERMINAL SWAP-RATE DRIFT COMPUTATIONS}

We apply our results on cross-variation derivatives ${ }^{1}$. In the case of a swap-rate, we have

$$
\mathrm{SR}_{j}=\frac{P_{j}-P_{n}}{A_{j}} .
$$

If we adopt $P_{N}$ as numeraire, we conclude that the drift of $\mathrm{SR}_{j}, \mu_{j}$ satisfies

$$
\mu_{j}=-\frac{P_{N}}{A_{j}}\left\langle A_{j} / P_{N}, \mathrm{SR}_{j}\right\rangle .
$$

We therefore need to evaluate this cross-variation term.

We now specialize to the case where $N=n$, we will return to the general case further down. We can write

$$
d \mathrm{SR}_{j}=\mathrm{SR}_{j} \sum_{k=1}^{F} a_{j k} d W_{k}+\mathrm{drift}
$$

where the Brownian motions, $W_{k}$, are independent. Clearly, we have

$$
\left\langle\mathrm{SR}_{j}, A_{j} / P_{n}\right\rangle=\mathrm{SR}_{j} \sum_{k=1}^{F} a_{j k}\left\langle W_{k}, A_{j} / P_{n}\right\rangle .
$$

This section was heavily influenced by unpublished work of Jochen Theis. 
If we can compute $\left\langle W_{k}, A_{j} / P_{n}\right\rangle$ for all $j$ and $k$ then we are done, and it will take $O(n F)$ computations to convert to drifts for $\mathrm{SR}_{j}$.

We now address how to compute

$$
\left\langle W_{k}, A_{j} / P_{n}\right\rangle
$$

for a fixed $k$ and all $j$ with order $n$ computations. We work backwards. The first case is $j=n-1$, where $A_{n-1} / P_{n}=\tau_{n-1}$ and the cross-variation is zero. Now suppose we have computed $\left\langle W_{k}, A_{j+1} / P_{n}\right\rangle$, we have

$$
\left\langle W_{k}, A_{j} / P_{n}\right\rangle=\left\langle W_{k}, P_{j+1} / P_{n}\right\rangle \tau_{j}+\left\langle W_{k}, A_{j+1} / P_{n}\right\rangle,
$$

the second term we already know. The first term we can rewrite:

$$
\begin{aligned}
\left\langle W_{k}, P_{j+1} / P_{n}\right\rangle & =\left\langle W_{k}, 1+\mathrm{SR}_{j+1} A_{j+1} / P_{n}\right\rangle, \\
& =\left\langle W_{k}, \mathrm{SR}_{j+1} A_{j+1} / P_{n}\right\rangle, \\
& =\left\langle W_{k}, \mathrm{SR}_{j+1}\right\rangle A_{j+1} / P_{n}+\left\langle W_{k}, A_{j+1} / P_{n}\right\rangle \mathrm{SR}_{j+1} .
\end{aligned}
$$

The first angle bracket is $\mathrm{SR}_{j+1} a_{j+1, k}$ by definition and the second is already known. This means that we can deduce the $j$ th term from the preceding computations with a fixed finite number of computations, and we are done.

Note that the above computations have computed the drift of $\mathrm{SR}_{j}$ whereas we would typically evolve $\log \left(\mathrm{SR}_{j}\right)$ instead, and we would therefore not carry out the final multiplication in (6.3), but subtract the standard $-0.5 C_{j j}$ for the transformation to log space.

We have computed the drift when $P_{n}$ is numeraire. We may wish to use another numeraire, we can compute the new drift using (4.11). We have

$$
\mu_{\mathrm{SR}_{j}, P_{N}}=\mu_{\mathrm{SR}_{j}, P_{n}}-\left\langle\mathrm{SR}_{j}, \frac{P_{n}}{P_{N}}\right\rangle \frac{P_{N}}{P_{n}} .
$$

We can expand $\mathrm{SR}_{j}$ in terms of $W_{k}$ as before, and we therefore need to compute

$$
\begin{aligned}
\frac{P_{N}}{P_{n}}\left\langle W_{k}, \frac{P_{n}}{P_{N}}\right\rangle & =-\frac{P_{N}}{P_{n}}\left\langle W_{k}, \frac{P_{N}}{P_{n}}\right\rangle \frac{P_{n}^{2}}{P_{N}^{2}} \\
& =-\left\langle W_{k}, \frac{P_{N}}{P_{n}}\right\rangle \frac{P_{n}}{P_{N}} .
\end{aligned}
$$

As $A_{N-1}-A_{N}=\tau_{N-1} P_{N}$, this is easily rolled into our original computation, and we are still within order $n F$ computations. 


\section{CONSTANT MATURITY MARKET MODELS}

Since short terms interests can vary a lot, a coupon which is linked to a short term LIBOR rate can change greatly from year to year. Products have therefore been introduced which have coupons that are linked to long swap-rates, since long rates tend to be more stable than short ones. Thus, for example, one may have a bond that pays a coupon which is a function of the ten-year swap rate on the coupon date. Such products are called constant maturity products or CMS products. One therefore wishes to be able to specify the dynamics of the ten-year swap rate starting on each coupon date for maximal effectiveness of the model. The coupon may also be a function not just of the current tenyear swap rate but also the previous coupon. For example, a CMS TARN struck at $K$ pays a multiple of $\max \left(K-2 \mathrm{SR}_{10}, 0\right)$ until a total predetermined coupon has been reached. It is then important not just to specify the dynamics of individual rate but also their joint dynamics and the constant maturity market model makes this possible.

In this section, we examine constant maturity market models. For a constant maturity model, we consider the set of rates

$$
\mathrm{SR}_{\alpha, \alpha+r}
$$

for a fixed $r$, and we make the convention that if $\alpha+r \geq n$, then we take it to equal $n$. We similarly let $A_{\alpha, \alpha+r}$ denote the annuity of $\mathrm{SR}_{\alpha, \alpha+r}$.

Note that we obtain a different rate for $\alpha=0,1, \cdots, n-1$, and that for the last $r$ rates we are working with co-terminal rates, and for those rates any analysis carries directly over from the co-terminal swap-rate market model. We need to compute drifts and find an algorithm for obtaining the bond ratios from the rates. We work with $P_{n}$ as numeraire and work backwards.

We have

$$
\mathrm{SR}_{j, r+j}=\frac{P_{j}-P_{r+j}}{A_{j, r+j}}
$$

by definition, (even when $r+j>n$ ), which implies

$$
\frac{P_{j}}{P_{n}}=\frac{P_{r+j}}{P_{n}}+\frac{A_{j, r+j}}{P_{n}} \mathrm{SR}_{j, r+j} .
$$

It is now clear that we can induct backwards computing $A_{j, r+j}$ and $P_{j} / P_{n}$ as we go.

If we are working in an $F$ factor model, as before, we can write

$$
d \mathrm{SR}_{j, r+j}=\mathrm{SR}_{j, r+j} \sum_{k=1}^{F} a_{j k} d W_{k},
$$

up to drift terms, and it follows that the drift of $\mathrm{SR}_{j, r+j}$ is equal to

$$
-\sum_{k=1}^{F} a_{j k} \frac{P_{n}}{A_{j, r+j}} \mathrm{SR}_{j, r+j}\left\langle W_{k}, \frac{A_{j, r+j}}{P_{n}}\right\rangle .
$$


If we can compute the quadratic variation terms with order $n F$ computations then that will be sufficient to show that we can compute all the drifts with that computational order. We work backwards. Suppose we know

$$
\begin{aligned}
& \left\langle\frac{A_{j, j+r}}{P_{n}}, W_{k}\right\rangle \text { for } j>l, \text { and } \\
& \left\langle\frac{P_{r}}{P_{n}}, W_{k}\right\rangle \text { for } r>l+1,
\end{aligned}
$$

we show that we can find

$$
\begin{aligned}
& \left\langle\frac{A_{l, l+r}}{P_{n}}, W_{k}\right\rangle \text { and } \\
& \left\langle\frac{P_{l+1}}{P_{n}}, W_{k}\right\rangle
\end{aligned}
$$

with a fixed number of computations, which will be sufficient. With knowledge of the second term, the first follows immediately from linearity and the values of $\left\langle\frac{A_{l+1, l+r+1}}{P_{n}}, W_{k}\right\rangle$, and $\left\langle\frac{P_{l+r+1}}{P_{n}}, W_{k}\right\rangle$.

We compute

$$
\left\langle\frac{P_{j+1}}{P_{n}}, W_{k}\right\rangle=\left\langle\mathrm{SR}_{j+1, r+j+1} \frac{A_{j+1, r+j+1}}{P_{n}}, W_{k}\right\rangle+\left\langle\frac{P_{j+r+1}}{P_{n}}, W_{k}\right\rangle .
$$

Using equation (7.1) and expanding, this is equal to

$$
\begin{aligned}
\mathrm{SR}_{j+1, r+j+1}\left\langle\frac{A_{j+1, r+j+1}}{P_{n}}, W_{k}\right\rangle & +\frac{A_{j+1, r+j+1}}{P_{n}}\left\langle\mathrm{SR}_{j+1, r+j+1}, W_{k}\right\rangle \\
& +\left\langle\frac{P_{j+r+1}}{P_{n}}, W_{k}\right\rangle .
\end{aligned}
$$

The first and third terms are known, and the second is trivial; we are done.

\section{CO-INITIAL SWAP-RATES}

As well as products depending on a single constant-maturity swap-rate, products exist which pay a function of the spread between two rates. Thus we may receive the difference of the ten-year rate and the two-year rate capped and floored. To deal with such products, the co-initial swap-rate market was introduced in Gallucio and Hunter (2004). We can solve it using similar techniques to the other cases we have discussed. 
For this section, we use $\mathrm{SR}_{j}$ to denote the swap-rate which was $\mathrm{SR}_{0, j}$ in Section 2, similarly for $A_{j}$. Our class of swap-rates is $\mathrm{SR}_{j}$ for $j=1, \cdots, n$, and they therefore all start on the same date but finish on varying dates. As usual, we must first develop an algorithm to deduce the bond ratios from the swap-rates. We will work with $P_{0}$ as numeraire in this section. In order to ease the notation, we shall also use a tilde to denote that a price has been divided by $P_{0}$. We thus have

$$
\begin{gathered}
\tilde{P}_{k}=\frac{P_{k}}{P_{0}}, \\
\tilde{A}_{j}=\frac{A_{j}}{P_{0}} .
\end{gathered}
$$

As

$$
\mathrm{SR}_{j}=\frac{P_{0}-P_{j}}{A_{j}},
$$

we see that

$$
\begin{aligned}
\tilde{P}_{j} & =1-\tilde{A}_{j} \mathrm{SR}_{j} \\
& =1-\tilde{A}_{j-1} \mathrm{SR}_{j}-\tau_{j-1} \tilde{P}_{j} \mathrm{SR}_{j},
\end{aligned}
$$

and hence that

$$
\tilde{P}_{j}=\frac{1-\tilde{A}_{j-1} \mathrm{SR}_{j}}{1+\tau_{j-1} \mathrm{SR}_{j}} .
$$

Inducting on $j$ increasing, it is clear how to deduce the bond ratios in order $n$ steps. By the usual arguments, to compute drifts for log-normal co-initial rates we need to find the cross-variation of $W_{k}$ and $\tilde{P}_{j}$, which equals

$$
\begin{aligned}
\left\langle W_{k}, \tilde{P}_{j}\right\rangle= & \left\langle W_{k}, \frac{1-\tilde{A}_{j-1} \mathrm{SR}_{j}}{1+\tau_{j-1} \mathrm{SR}_{j}}\right\rangle \\
= & \frac{\left\langle W_{k}, 1-\tilde{A}_{j-1} \mathrm{SR}_{j}\right\rangle}{1+\tau_{j-1} \mathrm{SR}_{j}}+\left\langle W_{k}, \frac{1}{1+\tau_{j-1} \mathrm{SR}_{k}}\right\rangle\left(1-\tilde{A}_{j-1} \mathrm{SR}_{j}\right), \\
= & -\frac{\left\langle W_{k}, \tilde{A}_{j-1}\right\rangle \mathrm{SR}_{j}}{1+\tau_{j-1} \mathrm{SR}_{j}}-\left\langle W_{k}, \mathrm{SR}_{j}\right\rangle \frac{\tilde{A}_{j-1}}{1+\tau_{j-1} \mathrm{SR}_{j}} \\
& -\left\langle W_{k}, 1+\tau_{j-1} \mathrm{SR}_{j}\right\rangle \frac{1-\tilde{A}_{j-1} \mathrm{SR}_{j}}{\left(1+\tau_{j-1} \mathrm{SR}_{j}\right)^{2}} \\
= & -\frac{\left\langle W_{k}, \tilde{A}_{j-1}\right\rangle \mathrm{SR}_{j}}{1+\tau_{j-1} \mathrm{SR}_{j}}-\frac{\left\langle W_{k}, \mathrm{SR}_{j}\right\rangle \tilde{A}_{j-1}}{1+\tau_{j-1} \mathrm{SR}_{j}} \\
& -\left\langle W_{k}, \mathrm{SR}_{j}\right\rangle \frac{\tau_{j-1}\left(1-\tilde{A}_{j-1} \mathrm{SR}_{j}\right)}{\left(1+\tau_{j-1} \mathrm{SR}_{j}\right)^{2}}
\end{aligned}
$$


If we make it our inductive hypothesis that we have already computed $\left\langle W_{k}, \tilde{P}_{j-1}\right\rangle$, and $\left\langle W_{k}, \tilde{A}_{j-1}\right\rangle$, it is clear that we can do the next term with a fixed finite number of computations, and the drifts follow as before.

\section{INCREMENTAL MARKET MODELS}

We have studied four cases: the LIBOR market model, the co-terminal swaprate market model, the co-initial swap-rate market model, and the constant maturity market model. For each of these, we have seen that the bond ratios can be deduced in order $n$ operations and the drifts computed in order $n F$ operations. One would, of course, like to formulate a general result. In this section, we introduce a new class of models for which we can compute the bond ratios with order $n$ multiplications, and the drifts with order $n F$ multiplications but both requiring order $n^{2}$ additions and subtractions. Additions are much faster in most architectures than multiplications so this is still a worthwhile result. We then see how adding a further additional hypothesis can reduce the total number of computations to order $n F$.

Any market model is determined by picking a set of times $t_{0}<t_{1}<\cdots<t_{n}$, and then choosing a subset of the swap-rates associated to (usually) contiguous subsets of those times. Let $P_{r}$ be value of the discount bond expiring at time $t_{r}$. We have

$$
\mathrm{SR}_{\alpha, \beta}=\frac{P_{\alpha}-P_{\beta}}{\sum_{r=\alpha}^{\beta-1} \tau_{r} P_{r+1}} .
$$

Specifying a market model is therefore equivalent to specifying two sequences in $\{0,1, \cdots, n-1\}: \alpha_{0}, \cdots, \alpha_{n-1}$, and $\beta_{0}, \cdots, \beta_{n-1}$, such that $\beta_{j} \geq \alpha_{j}+1$. Of course, for a given choice of the sequences, one needs to show that the bond ratios are uniquely determined.

Definition 9.1. A market model is incremental if $\beta_{0}=\alpha_{0}+1$, and for $j>0$, either

or

$$
\alpha_{j}=\min _{r<j} \alpha_{r}, \text { and } \beta_{j}=1+\max _{r<j} \beta_{r}
$$

$$
\alpha_{j}=-1+\min _{r<j} \alpha_{r}, \text { and } \beta_{j}=\max _{r<j} \beta_{r} .
$$

In other words, in an incremental market model the introduction of each new rate causes dependency on exactly one more discount bond. Note that as it is really bond ratios we care about, this is true even of $\mathrm{SR}_{\alpha_{0}, \beta_{0}}$, which depends purely on the ratio $P_{\alpha_{0}} / P_{\beta_{0}}$. If we fix a numeraire, there are $n$ bond ratios, and $n$ rates so when we get to the last rate we will have introduced dependency on all the bond ratios. 
Theorem 9.1. In an incremental market model, for any $N$ the bond ratios $P_{j} / P_{N}$ are determined by the swap-rates and can be deduced with order $n$ multiplications and order $n^{2}$ additions.

Proof. We take $N=\beta_{0}$. Once $P_{j} / P_{\beta_{0}}$ is known for all $j$, one simply writes

$$
\frac{P_{j}}{P_{N}}=\frac{P_{j}}{P_{\beta_{0}}} \frac{P_{\beta_{0}}}{P_{N}}
$$

to get the general case with an extra order $n$ computations.

We have

$$
\mathrm{SR}_{\alpha_{0}, \beta_{0}}=\frac{P_{\beta_{0}}-P_{\alpha_{0}}}{\tau_{\alpha_{0}}-P_{\beta_{0}}}
$$

so the ratio $\frac{P_{\alpha_{0}}}{P_{\beta_{0}}}$ is clearly determined.

We now show that given the bond ratios for the bonds underlying the first $r-1$ rates, we can deduce the extra bond ratio underlying $\mathrm{SR}_{\alpha_{r}, \beta_{r}}$ from its value. There are two cases corresponding to whether the new bond is at the beginning or end.

If it is at the end, we have $\beta_{r}=1+\max _{l<r} \beta_{l}$, and

$$
\mathrm{SR}_{\alpha_{r}, \beta_{r}}=\frac{P_{\alpha_{r}}-P_{\beta_{r}}}{\sum_{l=\alpha_{r}}^{\beta_{r}-1} \tau_{l} P_{l+1}} .
$$

Rearranging, we obtain

$$
\frac{P_{\beta_{r}}}{P_{\beta_{0}}}=\frac{\frac{P_{\alpha_{r}}}{P_{\beta_{0}}}-\sum_{l=\alpha_{r}}^{\beta_{r}-2} \tau_{l} \frac{P_{l+1}}{P_{\beta_{0}}} \mathrm{SR}_{\alpha_{r}, \beta_{r}}}{1+\tau_{\beta_{r}-1} \mathrm{SR}_{\alpha_{r}, \beta_{r}}} .
$$

The ratio is therefore determined. Similarly, if the new bond is at the beginning, we have

$$
\frac{P_{\alpha_{r}}}{P_{\beta_{0}}}=\frac{P_{\beta_{r}}}{P_{\beta_{0}}}+\mathrm{SR}_{\alpha_{r}, \beta_{r}} \sum_{l=\alpha_{r}}^{\beta_{r}-1} \tau_{l} \frac{P_{l+1}}{P_{\beta_{0}}}
$$

and the first ratio is determined.

How many computations will this take? At each stage, we store each new bond ratio and its multiplication by the appropriate accrual, $\tau_{l}$, it is then clear that we only need a fixed number of multiplication per step and therefore order $n$ in total. However, the sums will require up to $n$ additions per step 
so we have order $n^{2}$ additions in total. Note that in each of the four cases we studied in detail, there was extra structure that reduced the number of additions, but it seems unlikely that this will be possible in general without extra hypotheses.

By the same arguments as in previous sections, if we take $P_{\beta_{0}}$ as numeraire, we can deduce the drift of $\mathrm{SR}_{\alpha_{j}, \beta_{j}}$ for all $j$ from the knowledge of

$$
\left\langle W_{k}, \frac{A_{\alpha_{j}, \beta_{j}}}{P_{\beta_{0}}}\right\rangle,
$$

with order $n F$ operations. We proceed inductively on $j$ as usual and each stage store the cross-variation of the swap-rate ratio of the new bond to the numeraire and its value multiplied by the appropriate accrual.

Just as with the deduction of the bond ratios, we have to proceed differently according to whether the introduction of the new bond is at the beginning or the end of the known cases. If it is at the start, using (9.2), we have

$$
\begin{aligned}
\left\langle W_{k}, \frac{P_{\alpha_{r}}}{P_{\beta_{0}}}\right\rangle= & \left\langle W_{k}, \frac{P_{\beta_{r}}}{P_{\beta_{0}}}\right\rangle+\left\langle W_{k}, \mathrm{SR}_{\alpha_{r}, \beta_{r}}\right\rangle \sum_{l=\alpha_{r}}^{\beta_{r}-1} \tau_{l} \frac{P_{l+1}}{P_{\beta_{0}}} \\
& +\mathrm{SR}_{\alpha_{r}, \beta_{r}} \sum_{l=\alpha_{r}}^{\beta_{r}-1} \tau_{l}\left\langle W_{k}, \frac{P_{l+1}}{P_{\beta_{0}}}\right\rangle .
\end{aligned}
$$

This is computable with order $n$ additions and a fixed finite number of multiplications.

If at the end, using (9.1), we have

$$
\begin{aligned}
&\left\langle W_{k}, \frac{P_{\beta_{r}}}{P_{\beta_{0}}}\right\rangle=\left(1+\tau_{\beta_{r}-1} \mathrm{SR}_{\alpha_{r}, \beta_{r}}\right)^{-1} \\
&\left\langle\left\langle W_{k}, \frac{P_{\alpha_{r}}}{P_{\beta_{0}}}\right\rangle-\sum_{l=\alpha_{r}}^{\beta_{r}-2} \tau_{l}\left\langle W_{k}, \frac{P_{l+1}}{P_{\beta_{0}}}\right\rangle \mathrm{SR}_{\alpha_{r}, \beta_{r}}-\sum_{l=\alpha_{r}}^{\beta_{r}-2} \tau_{l} \frac{P_{l+1}}{P_{\beta_{0}}}\left\langle W_{k}, \mathrm{SR}_{\alpha_{r}, \beta_{r}}\right\rangle\right) \\
&+\frac{\tau_{\beta_{r}-1}\left\langle W_{k}, \mathrm{SR}_{\alpha_{r}, \beta_{r}}\right\rangle}{\left(1+\tau_{\beta_{r}-1} \mathrm{SR}_{\alpha_{r}, \beta_{r}}\right)^{2}}\left(\frac{P_{\alpha_{r}}}{P_{\beta_{0}}}-\sum_{l=\alpha_{r}}^{\beta_{r}-2} \tau_{l} \frac{P_{l+1}}{P_{\beta_{0}}} \mathrm{SR}_{\alpha_{r}, \beta_{r}}\right) .
\end{aligned}
$$

This can also be computed with order $n$ additions and a fixed finite number of multiplications.

Once we have the cross-variation derivative with each bond ratio, the crossvariation derivatives with the annuities are straightforward additions and we are done.

Studying the above proofs, one sees that the failure of the algorithm to attain order $n$ operations for the deduction of bonds-ratios and order $n F$ for the 
computation of drifts arises from the need to compute annuities. If we put an additional hypothesis on the annuities, we can attain these faster speeds.

Definition 9.2. We shall say that a class of market models is fully incremental of order $\theta$ if there exists $\theta$ independent of $n$ such that for each $j$, there exists $i<j$, such that $\mathrm{SR}_{i}$ differs from $\mathrm{SR}_{j}$ by at most $\theta$ bonds.

It is clear from studying the proofs above that the bond-ratios can be deduced in $O(n \theta)$, operations and the drifts in $O(n(F+\theta))$ operations. The constant maturity market model is fully incremental of order 2 , the other cases we have studied are fully incremental of order 1 .

\section{NUMERicAl RESUlts}

In this section, we present timings using these techniques. The purpose of the modelling was to demonstrate the behaviour as a function of the number of rates, $n$, rather than to do the fastest possible implementation. For each of the

\section{TABLE 1}

TIMINGS FOR EVOLVING A CONSTANT MATURITY SWAP MARKET MODEL OF CONSTANT MATURITY 4 FOR A 3-FACTOR MODEL WITH VARYING NUMBERS OF RATES. THE FINAL COLUMN DISPLAYS THE BEST FIT PARABOLA THROUGH THE POINTS.

\begin{tabular}{crr}
\hline \hline Rates & Time & \multicolumn{1}{c}{ Fit } \\
\hline 5 & 10.11 & 10.17 \\
10 & 31.97 & 31.77 \\
15 & 65.36 & 65.11 \\
20 & 110.68 & 110.19 \\
25 & 165.65 & 167.01 \\
30 & 236.24 & 235.57 \\
\hline \hline
\end{tabular}

TABLE 2

TIMINGS FOR EVOLVING A CONSTANT MATURITY SWAP MODEL OF CONSTANT MATURITY 4 FOR A 5-FACTOR MODEL WITH VARYING NUMBERS OF RATES. THE FINAL COLUMN DISPLAYS THE BEST FIT PARABOLA THROUGH THE POINTS.

\begin{tabular}{rrr}
\hline \hline Rates & Time & \multicolumn{1}{c}{ Fit } \\
\hline 5 & 12.41 & 13.02 \\
10 & 41.19 & 40.51 \\
15 & 85.63 & 85.01 \\
20 & 146.60 & 146.54 \\
25 & 223.33 & 225.09 \\
30 & 321.58 & 320.66 \\
\hline \hline
\end{tabular}


TABLE 3

TIMINGS FOR EVOLVING A CO-TERMINAL SWAP-RATE MARKET MODEL FOR A 3-FACTOR MODEL WITH VARYING NUMBERS OF RATES.

THE FINAL COLUMN DISPLAYS THE BEST FIT PARABOLA THROUGH THE POINTS.

\begin{tabular}{crr}
\hline \hline Rates & Time & \multicolumn{1}{c}{ Fit } \\
\hline 3 & 5.82 & 6.06 \\
5 & 12.69 & 12.49 \\
10 & 37.95 & 37.69 \\
15 & 75.74 & 75.93 \\
20 & 127.10 & 127.22 \\
25 & 191.69 & 191.55 \\
\hline \hline
\end{tabular}

TABLE 4

TIMINGS FOR EVOLVING A CO-TERMINAL SWAP-RATE MODEL FOR A 5-FACTOR MODEL WITH VARYING NUMBERS OF RATES.

THE FINAL COLUMN DISPLAYS THE BEST FIT PARABOLA THROUGH THE POINTS.

\begin{tabular}{rrr}
\hline \hline Rates & Time & \multicolumn{1}{c}{ Fit } \\
\hline 5 & 15.05 & 14.28 \\
10 & 46.53 & 46.44 \\
15 & 93.81 & 95.92 \\
20 & 165.20 & 162.73 \\
25 & 245.95 & 246.85 \\
\hline \hline
\end{tabular}

TABLE 5

TIMINGS FOR EVOLVING A CO-INITIAL SWAP-RATE MARKET MODEL FOR A 3-FACTOR MODEL WITH VARYING NUMBERS OF RATES.

THE FINAL COLUMN DISPLAYS THE BEST FIT LINE THROUGH THE POINTS.

\begin{tabular}{rcc}
\hline \hline Rates & Time & Fit \\
\hline 3 & 1.76 & 1.78 \\
5 & 2.14 & 2.14 \\
10 & 3.07 & 3.04 \\
15 & 3.96 & 3.95 \\
20 & 4.84 & 4.85 \\
25 & 5.76 & 5.75 \\
30 & 6.64 & 6.65 \\
\hline \hline
\end{tabular}

constant maturity and co-terminal cases, we step all the rates that have not reset to each of the reset dates. We show timings for a fixed number of factors. Since we carry out an order $n F$ algorithm for each of $n$ steps, we obtain timings that are parabolic in $n$, and we display the values of a fitted parabola 
TABLE 6

TIMINGS FOR EVOLVING A CO-INITIAL SWAP-RATE MODEL FOR A 5-FACTOR MODEL WITH VARYING NUMBERS OF RATES. THE FINAL COLUMN DISPLAYS THE BEST FIT LINE THROUGH THE POINTS.

\begin{tabular}{ccc}
\hline \hline Rates & Time & Fit \\
\hline 5 & 2.36 & 2.35 \\
10 & 3.35 & 3.33 \\
15 & 4.28 & 4.31 \\
20 & 5.27 & 5.28 \\
25 & 6.26 & 6.26 \\
30 & 7.25 & 7.24 \\
\hline \hline
\end{tabular}

through the timings in each case. The parabola is always a close fit, numerically confirming that the algorithm is second order in $n$. See tables 1 and 2 for the constant maturity case, and tables 3 and 4 for the co-terminal case. In each case, we use 32768 paths. The rates are yearly rates and we step to the reset time of each rate using the predictor-corrector algorithm. The simulations were run on a $400 \mathrm{MHz}$ PC.

In the co-initial case, we only evolve to the common initial time so we expect linear behaviour for speed. We display the timings and the best fit line through them in tables 5 and 6.

\section{CONCLUSION}

We have examined a number of special cases: the co-terminal swap-rate model, the co-initial swap-rate model, the constant maturity market model, as well as the more general case of the incremental market model. For these cases, we have shown that efficient algorithms exist for the evolution of time steps. These models are therefore equally attractive to the LIBOR market model in terms of efficiency and one should make model choice on the basis of other issues such as ease of calibration, and adaptation to the product being studied.

\section{REFERENCES}

Brace, A., Gatarek, D. and Musiela, M. (1997) The market model of interest-rate dynamics, Mathematical Finance 7, 127-155.

Brigo, D. and Mercurio, F. (2001) Interest Rate Models - Theory and Practice, Springer Verlag. Gallucio, S., Huang, Z., Ly, J.-M. and Scaillet, O. (2007) Theory of calibration of swap market models, Mathematical Finance 17(1), 111-141.

Gallucio, S. and Hunter, C. (2004) The Co-initial Swap Market Model, Economic Notes by Banca Monte dei Paschi di Siena SpA, 33(2), 209-232.

Hunter, C., JÄCKel, P. and Joshi, M. (2001) Getting the drift, Risk, July 2001. 
JAMSHIDIAN, F. (1997) LIBOR and swap market models and measures, Finance and Stochastics 1, 293-330.

Joshi, M. (2003) The concepts and practice of mathematical finance, Cambridge University Press. JoshI, M. (2003b) Rapid Drift Computations in the LIBOR market model, Wilmott, May 2003. JosHI, M. (2006) Achieving decorrelation and speed simultaneously in the LIBOR market model, the Journal of Risk 9(1), 147-153.

Musiela, M. and Rutowski, M. (1997) Martingale Methods in Financial Modelling, Springer Verlag.

Pietersz, R. and Van Regenmortel M. (2006) Generic Market Models, Finance and Stochastics 10, 507-528.

\section{MARK JOSHI}

Centre for Actuarial Studies,

Department of Economics,

University of Melbourne,

Victoria 3010,

Australia

E-mail:mark@markjoshi.com

\section{LORENZO LIESCH}

$U B M$,

Financial Risk,

Via Tommaso Grossi 10,

20141 Milan,

Italy

E-mail:lieschl@libero.it 